\title{
Anomalous zero-group-velocity photonic bonding states with local chirality
}

\author{
Moïse Sotto ${ }^{1}$, Kapil Debnath ${ }^{2,3}$, Ali Z. KhokhaR ${ }^{2}$, Isao Tomita ${ }^{1,4}$, David Thomson ${ }^{2}$, And \\ SHINICHI SAITO ${ }^{1, *}$ \\ ${ }^{1}$ Sustainable Electronic Technologies, Electronics and Computer Science, Faculty of Physical Sciences and Engineering, University of Southampton, University \\ Road, SO17 1BJ, Southampton, United Kingdom. \\ ${ }^{2}$ Optoelectronics Research Centre, Faculty of Physical Sciences \& Engineering, University of Southampton, Highfield Campus, Southampton, SO17 1BJ, \\ United Kingdom. \\ ${ }^{3}$ Department of Electronics \& Electrical Communication Engineering. Indian Institute of Technology Kharagpur, Kharagpur, West Bengal, India-721302. \\ ${ }^{4}$ Department of Electrical \& Computer Engineering, National Institute of Technology, Gifu College, Japan. \\ ${ }^{*}$ Corresponding author:S.Saito@soton.ac.uk
}

Photonic Crystal Waveguides (PCWs) are promising candidates for the basic building blocks of quantum information processing because they support circular polarization points that can unbalance the directionality of an integrated quantum emitter (QE). Nevertheless, the Purcell effect at circular polarization points saturates near the band-edge, the preferred region for Quantum ElectroDynamics (QED). Consequently, chirality and ultra-strong light-matter interaction are difficult to combine. Here, we detract from the vicinity of the band-edge, and couple modes with different parities by breaking the mirror symmetry. Using threedimensional finite-difference time domain method (3DFDTD), simulated bandstructures of the implemented Photonic Bonding States (PBS) display single-mode anomalous zero-group-velocity (ZGV) points far from the band edge. The electric field patterns of these points feature circular polarization points at high field intensity regions where a $Q E$ would acquire unidirectional emission behavior. Fabricated devices in Silicon (Si) slabs demonstrate the predicted coupling energy between the modes and the signature of singlemode anomalous $Z G V$ points. This method to engineer PBS in PCWs paves the way for outperforming chiral light-matter experiment on-chip. ๑ 2018 Optical Society of America

OCIS codes: (130.5296) Photonic crystal waveguides; (260.5430) Polarization; (270.5580) Quantum electrodynamics.

http://dx.doi.org/10.1364/ao.XX.XXXXXX

\section{INTRODUCTION}

There are great expectations that PCWs may serve as the platform of quantum information processing on-chip. Due to their photonic BandGap (BG)[? ], PCWs provide a drastic enhance- ment of the spontaneous emission of an embedded QE[? ? ? ? ]. This is attributed to the tight confinement and the slow-down effect $\left(n_{g}\right.$ the group index) of the light. Due to this, the decay rate of the $\mathrm{QE}$ into radiative modes outside of the platform is greatly suppressed. The Purcell factor $\left(P_{f}\right)$ is the figure of merit for the effect of the photonic environment on the spontaneous emission[? ? ? ]. In addition to the light-matter interaction enhancement, the BG confinement implies that Transverse Electric (TE) guided modes in PCWs possess both transverse and longitudinal electric field components. The phase relation between these components involves local chirality across the PCW unit cell which is characterized by circular polarization points in their field patterns[? ? ]. Therefore, an embedded QE with spindependent optical transitions at such a particular position infers a non-time-reversal symmetry to the coupled system, and directional factors $>90 \%$ have been experimentally demonstrated [? ]. However, conventional PCWs with mirror symmetry, as shown in Fig.??a, prevent the overlap of high field intensity regions and in-plane circular polarization points. Non-symmorphic PCWs like glide-plane waveguides [? ? ? ? ], where one side of the waveguides is shifted along the direction of propagation [Fig.??b], break this paradigm and enhance the electric field intensity of circular polarization points.

Despite the set of advantages conferred by PCWs, chiral lightmatter interaction using this platform is hampered by the selflimitation of utilizing the guided mode near the band-edge. Due to the fact that the chirality is vanishing everywhere in the vicinity of the Brillouin Zone (BZ) edge[? ], $P_{f}$ at circular polarization locations inherently saturates near this point[? ]. So far, glide-plane waveguides, engineered to unify local chirality and light-matter interaction, are constrained to either moderate $n_{g}$ values < 100[? ] (limiting the coupling) or multi-mode operation[? ] (annihilating the uni-directionality). Other methods are needed to force these properties to work in unison and overcome the competition between the vanishing of chirality and the increase of the slow light effect at the band-edge. One unexplored approach is to bring the slow-down effect inside the BZ. 
In this work, we propose to achieve such a regime by establishing single-mode ZGV points inside the BZ. The PCW is designed such as bands with different parities are intersecting below the light line and propagate in opposite direction. Thus, the break of the parity ensures branch exchange leading to anomalous ZGV points $\left(n_{g} \rightarrow \infty\right)$ where the chirality is prevented to vanish.

We start by presenting the wave properties of ZGV points and their consequences on the chiral light-matter interaction enhancement. We emphasize the substitution of the mirror symmetry with glide-plane axes and its impact on the bandstructure. A particular attention is paid to the emergence of anomalous ZGV points. Then, experimental observation of the PBS is achieved from fabricated Si PCWs. The coupling between the PBS as well as the signatures of the anomalous ZGV points are extracted and compared to the simulation results. In the following section, we investigate their local chirality at the anomalous ZGV points through the calculation of the Stokes parameters inside the PCW unit cell. There is a strong interest in the circular polarization resulting from the coupling because the studied even and odd modes have their dominant electric field components perpendicular to each other at the crossing point. In the conclusion, we summarize about the merit of bringing the slow-down effect inside the BZ.

This work establishes the foundation for out-performing future QED experiments on-chip combining a divergent photonic local density of states with extended local chirality.

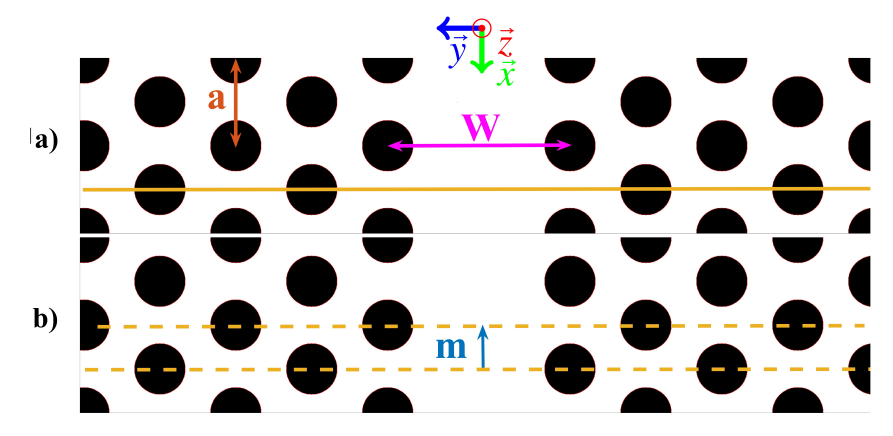

Fig. 1. Schematic of the PCWs with lattice constant $a$, the waveguide width $\mathrm{W}$ (i.e. the distance between the center of the first circular hole rows). The figures introduce the definition of the mismatch $m$ between both parts of the PhC regions. (a) For no mismatch, the structure can be constructed with one side and its mirror image by the plane $(\widehat{x O z})$ placed at $y=0$. (b) For a mismatch $m$, one side has been translated by a distance $m$ along the propagation axis. Presented here is the maximum mismatch $m / a=0.5$.

\section{THE EXTENT OF CIRCULAR POLARIZATION AT ZGV POINTS}

For a TE modes in PCWs as shown in Fig.??, the electric field components of a circular polarization point obey the relation:

$$
E_{x}= \pm i E_{y}
$$

where $E_{x}\left(E_{y}\right)$ is the longitudinal(transverse) electric field component, while $+(-)$ stands for fully right(left)-handed polarization. Nevertheless, the extent of the circular polarization strongly depends on the wave-vector of the guided modes. It has a significant importance on the prominence of these points, especially near the boundary of the Brillouin Zone (BZ). Due to the periodicity and the time reversal symmetry of the Maxwell equations, it follows that at the band-edge $(k=\pi / a)$, field envelops $\Psi$ can be transformed as:

$\Psi_{\pi / a}(x, y, z)=\Psi_{-\pi / a}^{*}(x, y, z)=\Psi_{-\pi / a+2 \pi / a}^{*}(x, y, z)=\Psi_{\pi / a}^{*}(x, y, z)$,

which states that the fields envelops are real at this high symmetry point. On the other hand, the light-matter interaction at the position $\vec{r}=(x, y, z)$, characterized by $P_{f}$, is directly proportional to the local density of photonic states[? ]:

$$
P_{f}(\vec{r}) \propto\left|n_{g}\right| E^{2}(\vec{r}),
$$

where the electric field is normalized by $\int_{\text {unit }} \epsilon(\vec{r}) \vec{E}^{2}(\vec{r}) d \vec{r}=1$. The slow-down effect at the band-edge is coming from the interference between backward and forward wave that have the same envelop. This standing wave characteristic states what impedes the use of the band-edge for chiral QED experiments, circular polarization locations, denoted $\vec{r}_{c}$, do not exist for this wave-vector. In consequence, the local density of photonic states of these points reach its maximum before the group velocity diverges and the asymptotic behavior of $\left|n_{g}\right| E^{2}\left(\vec{r}_{c}\right)$ in the vicinity of the BZ implies that[? ? ]:

$$
\lim _{k \rightarrow \frac{\pi}{a}} P_{f}\left(\vec{r}_{c}\right) \rightarrow 0
$$

On the other hand, for a wave-vector inside the Brillouin zone $(0<K<\pi / a)$, the Bloch theorem,

$$
\Psi_{K}(x+a, y, z)=\Psi_{K}(x, y, z) e^{-i K a},
$$

implies that the field envelop must be complex due to the phase shift caused by a translation of one period regardless of the group velocity. In the specific case where the group velocity of this mode is zero, it is well known to be an anomalous ZGV points. Such points are of special interest as their properties detract from general standing wave characteristics[? ? ? ], because their wave-vectors are not associated with a high symmetry point in the BZ. Only such a mode allows the local density of photonic states of circular polarization points $\left|n_{g}\right| E^{2}\left(\vec{r}_{c}\right)$ to diverge. However, it is important that these anomalous ZGV points are single-mode (i.e. unique in the frequency regime) in order to ensure chiral behavior. Previous work showed that multi-mode operation annihilated chirality. This is due to the contributions of different wave-vectors for which opposite group velocities and/or opposite circular polarizations are destructively interfering with each others to degrade the local chirality[? ]. In the following sections the implementation and the identification of single-mode anomalous ZGV points inside the telecom bandwidth are presented.

\section{BANDSTRUCTURE OF THE PHOTONIC BONDING STATES}

The method chosen to implement single-mode anomalous ZGV points is to couple modes of different symmetries for which dispersion relations cross within the photonic BG and below the light line. The Photonic Crystal (PhC) studied here is a triangular lattice with a lattice constant $a=450 \mathrm{~nm}$, patterned with circular holes of radius $r=0.29 a$. The PhC slab is a $220 \mathrm{~nm}$ thick $\mathrm{Si}$ membrane. The width of the waveguide (the center-to-center distance of the first rows of holes) is chosen to be $W=1.2 a \sqrt{3}$. 
The photonic bandstructure of the TE guided modes has been calculated by 3D-FDTD. In the symmorphic waveguide [Fig.??a], the bands have been plotted in Fig.?? as solid and dashed black lines for odd and even modes respectively. Due to their different parities under mirror symmetry,

$$
\hat{R}_{y} E_{y}(x, y, z)= \pm E_{y}(x,-y, z)
$$

where $\hat{R}_{y}$ is the reflection operator and $+(-)$ stands for even(odd) parity, these modes are independent and the lowest energy pair of modes intersects at the wave-vector $k_{0}=0.3075(2 \pi / a)$. In order to couple the modes, one side of the $\mathrm{PhC}$ is shifted with respect to the other along the propagation direction by the distance $m$ [Fig.??b]. The bandstructures have been calculated for the so - called mismatch parameter ' $m / a^{\prime}$ from 0 to 0.5 in steps of 0.125 [Fig.??]. The colored curves correspond to the PBS es-

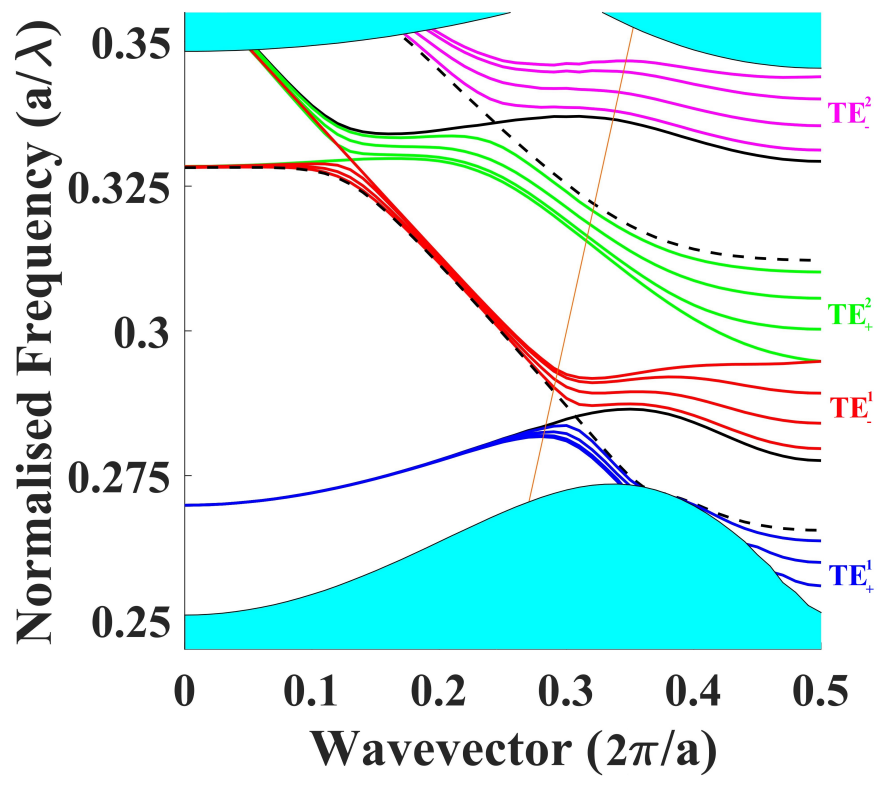

Fig. 2. The photonic bandstructure of the TE guided modes PCW with the parameters $\mathrm{a}=450 \mathrm{~nm}, \mathrm{~W}=1.2 \sqrt{3} a$ for different mismatches between PhC sides. For $m=0$, odd and even modes are respectively represented in dashed and solid black lines. Whereas for $m / a=0.125,0.25,0.375$ and 0.5 , the $\mathrm{TE}_{1}^{+}, \mathrm{TE}_{1}^{-}, \mathrm{TE}_{2}^{+}$ and $\mathrm{TE}_{2}^{-}$modes are represented in blue, red, green and purple respectively. The coupling is increasing with $m / a$ and the bonding states are repelled away from each other. The light line is represented in orange and the PhC slab modes in cyan.

tablished by $m / a \neq 0$. Due to the break of mirror symmetry, the bands transit from crossing (between odd and even modes) to anti-crossing behavior (between $\mathrm{TE}_{1(2)}^{+}$and $\mathrm{TE}_{1(2)}^{-}$modes). The lowest energy PBS pair will be the principal focus of this study. The repulsive coupling strength between the PBS is increasing with $m / a$. At the crossing point $\left(k_{0}\right)$, odd and even modes propagate at the same frequency, but with opposite sign group velocities. Consequently, the branch exchange leads to ZGV points at anomalous positions in the BZ, by imposing the $\mathrm{TE}_{1}^{+}$mode to transit from a forward propagating to a backward propagating wave, at the avoided crossing point (and vice versa for the $\mathrm{TE}_{1}^{-}$mode).

Along with the energy splitting, the wave-vectors of the ZGV points do not stand at $k_{0}$. This is attributed to the dispersion of the even and odd modes are which not equal at $k_{0}$ $\left(\left|n_{g, \text { odd }}\right|\left(k_{0}\right) \neq\left|n_{g, \text { even }}\right|\left(k_{0}\right)\right)$. Thus, the anomalous ZGV points of each bonding band also repulse each other along the propagation constant axis. Nevertheless, this artefact has no consequences for using the PCW studied here in QED experiments. Because the anomalous ZGV points, which will be labeled as $k_{0}^{+}$and $k_{0}^{-}$ for the $\mathrm{TE}_{1}^{+}$and $\mathrm{TE}_{1}^{-}$modes respectively, stay below the light line, regardless of $m / a$.

Although the parity is a discrete symmetry, an analogy can be drawn between the coupling phenomenon induced by the mismatch and the coupling of guided modes with same parity[? ? ] forced by the periodicity (lack of continuous translational symmetry). In symmorphic PCWs, the order of the guided mode can change from one to another across the same photonic band[? ? ]. This is generally accompanied by a sharp transition of the dispersion behavior[? ? ? ]. This effect has been extensively used to tailor the dispersion relation and engineer a dispersionless (constant $n_{g}$ ) bandwidth. By controlling the modal confinement through slight modifications (i.e. the size of the holes of the nearest neighboring rows[? ] or their positions[? ]), the distribution of the mode orders along the bands is thus altered. Here, the replacement of the mirror symmetry by glide-plane axes implies fortuitous intersections of modes with different parities to split rather the coupling of modes with same parity induced by the folding the bandstructure (i.e. periodicity).

In addition to being repulsed by the $\mathrm{TE}_{1}^{+}$mode, the $\mathrm{TE}_{1}^{-}$mode is attracted by the $\mathrm{TE}_{2}^{+}$mode at the band-edge. For $m / a=0.5$, these two modes merge together. As explained in Section I, the field must be absolutely real at the band-edge, regardless of the symmetry class group. Because the structure is invariant by the glide-plane operation $\hat{G}_{m}$, it implies that the electric field $\vec{E}_{\vec{k}}$ is an eigenstate of this transformation:

$$
\hat{G}_{m} \vec{E}_{\vec{k}}=\hat{T}_{x=m} \hat{R}_{y} \vec{E}_{\vec{k}^{\prime}}
$$

where $\hat{T}_{x=m}$ is the translation by $m$ along the propagation axis. Because applying twice the glide-plane axis symmetry is equivalent to a translation of a lattice period, it follows that at the band-edge, for $m / a=0.5$ :

$$
\vec{E}_{\pi / a}(x+a / 2,-y, z)= \pm i \vec{E}_{\pi / a}(x, y, z),
$$

which inhibits the realness of the electric field envelop. To ensure the time reversal property of the fields guided by this wavevector, a second mode must merge to counterbalance this extraphase and satisfies:

$$
\vec{E}_{1, \pi / a}=\vec{E}_{2, \pi / a}^{*} .
$$

Therefore, all the modes merge in pairs at the band-edge to become two-fold degenerate.

Regarding the single-mode operation of the anomalous ZGV points, it can be noted that: $\max \left(\lambda_{\text {odd }}^{-1}\right)>\lambda^{-1}\left(k_{0}\right)>\lambda_{\text {odd }}^{-1}(\pi / a)$, because the odd mode is not monotonic. This implies that anomalous ZGV points are not unique at their frequencies until $m / a$ crosses certain thresholds. These conditions, related to the repulsion between $\mathrm{TE}_{1}^{+}$and $\mathrm{TE}_{1}^{-}$and the attraction between $\mathrm{TE}_{1}^{-}$ and $\mathrm{TE}_{2}^{+}$, will be thoroughly explored in the following section with a finer scan of $m / a$.

We have shown that a fine tuning of the glide-plane symmetry allowed the engineering of confined anomalous ZGV points, by coupling pairwise degenerate modes without a structural potential barrier. If band repulsion has already been theoretically studied in glide-plane-waveguides[? ] and experimentally observed in a contra-directional coupler[? ], both occurred above the light-line. Our work sets a completely new stage for the practical application of propagating PBS with anomalous ZGV points that are truly confined. 

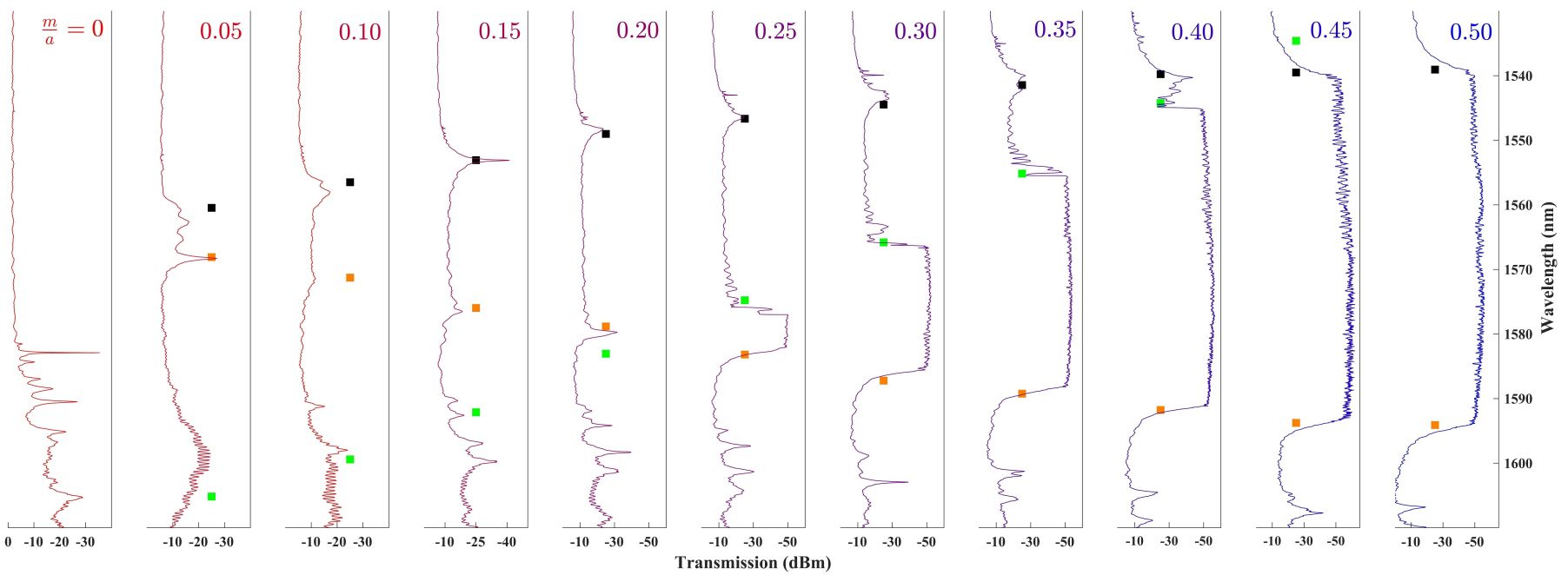

Fig. 3. Normalized transmission spectra of the waveguides with different ( $m / a)$ values from 0 (red) to 0.5 (blue) in steps of 0.05 . The simulated wavelengths of the anomalous ZGV points (orange and black for bonding and anti-bonding at $k_{0}^{+}$and $k_{0}^{-}$respectively) has been superimposed onto the measurements as well as the frequency of the anti-bonding mode at the end of the BZ (in green).

\section{SPECTROSCOPY OF THE ANOMALOUS ZGV POINTS}

PCWs with various mismatches between the 50 period long $\mathrm{PhC}$ regions were fabricated on a SOI wafer. Thereupon, the transmission properties of the PBS in the different PCWs were measured using a fiber coupler set-up. Both the fabrication process and the measurement set-up details have been reported in the Appendix. Here, we report the results of the experiment in Fig.??. The value of $m / a$ is scanned from 0 to 0.5 in steps of 0.05 to explore the branch exchange by means of the emergence of the ZGV points. The simulated wavelengths of the $\mathrm{TE}_{1}^{+}$mode at $k_{0}^{+}$and $\mathrm{TE}_{1}^{-}$mode at $k_{0}^{-}$have been superimposed on the measurements in orange and black respectively. The wavelength of the $\mathrm{TE}_{1}^{-}$mode at the band-edge has also been plotted in green. The points are blue-shifted approximately $0.46 \%$ in wavelength to overlap the experimental values.

Due to the peculiar dispersion relation of the odd mode, explained in the previous section, the anomalous ZGV points take two different signatures in the transmission spectra, depending on if they are single-mode or not. The transition between multimode to single-mode operation of the $T E_{1}^{+}$and $T E_{1}^{-}$modes is emphasized by the change of regime in the transmission spectra. The anomalous ZGV points are associated with the limits of the sub-BG opening with $m / a$, when they are single-mode. On the other hand, ZGV points are represented by clear local drops of the transmission when they are multi-mode due to the non-zero density of state on both side of these points. However, they still appear in a distinctive manner among the transmission bandwidth due to the multi-mode interference at this precise frequency. As observed on the transmission spectra, this effect is salient due to the ZGV nature of the PBS modes at $k_{0}^{+}$and $k_{0}^{-}$[? ]. For example, at $m / a=0.15$ the propagation losses of the $\mathrm{TE}_{1}^{-}$mode at $k_{0}^{-}$are $25 \mathrm{dDm}$ higher than the surrounding wavelengths. This drastic increase of the optical losses on a such narrow bandwidth is assigned to the raise of the group index as a ZGV points is approached[? ]. Because the effective coupling strength between the PBS is increasing with $m / a$, the drops in transmission at $k_{0}^{+}$and $k_{0}^{-}$are indeed repulsing from each other, as predicted by the FDTD simulations. The sub-BG only opens when the attraction between $\mathrm{TE}_{1}^{-}$and $\mathrm{TE}_{2}^{+}$at the band-edge allows it. This implies that the bending of $\mathrm{TE}_{1}^{-}$band induces two different thresholds:

The $\mathrm{TE}_{1}^{+}$mode at $k_{0}^{+}$becomes single-mode and the lower limit of the sub-BG for $m / a \geq 0.25$ while the $\mathrm{TE}_{1}^{-}$mode at $k_{0}^{-}$is singlemode and the upper limit of the sub-BG for $m / a \geq 0.45$.

These transitions from the dips to the limits of the sub-BG predicted by the calculations (colored points) are matching the experimental values.

This spectroscopy experiment highlights the powerful potential and the reliability of the method presented here to implement PBS. The scan of the mismatch parameter allowed to observe the repulsion between the PBS predicted in the previous section and the induced strong energy splitting was confirmed and measured to be $6.74 \mathrm{THz}$ centered at $191.38 \mathrm{THz}$ for $m / a=0.5$. The conditions on the mismatch for the anomalous ZGV points to be single-mode have been established and agree with FDTD simulations.

\section{LOCAL CHIRALITY OF THE PBS}

The mismatch does not only introduce dispersion singularities, as shown in the previous sections, but also profoundly alters the electric field profiles. This is especially true for the anomalous ZGV points where the modes transit from one order to another. The wave-functions of the PBS are a combination of the even and odd modes[? ] which have perpendicular dominant electric field components (transverse and longitudinal respectively). This constitutes a relevant interest to investigate the resulting polarization of the anomalous ZGV points, particularly in the non-symmorphic structure where high field intensity regions can be circularly polarized. To our knowledge, the effect of anti-crossing bands on the field ellipticity was never explored in previous analysis of non-symmorphic waveguides.

Here we characterized the enhancement of the chirality extent with respect to $m / a$. In order to point out the local chirality of the anomalous ZGV points, the Stokes parameters[? ] $S_{0}$ and $S_{3}$ of the $\mathrm{TE}_{1}^{+}$and $\mathrm{TE}_{1}^{-}$modes at $k_{0}^{+}$and $k_{0}^{-}$respectively, have been mapped inside the unit cell of the non-symmorphic PCWs. The 

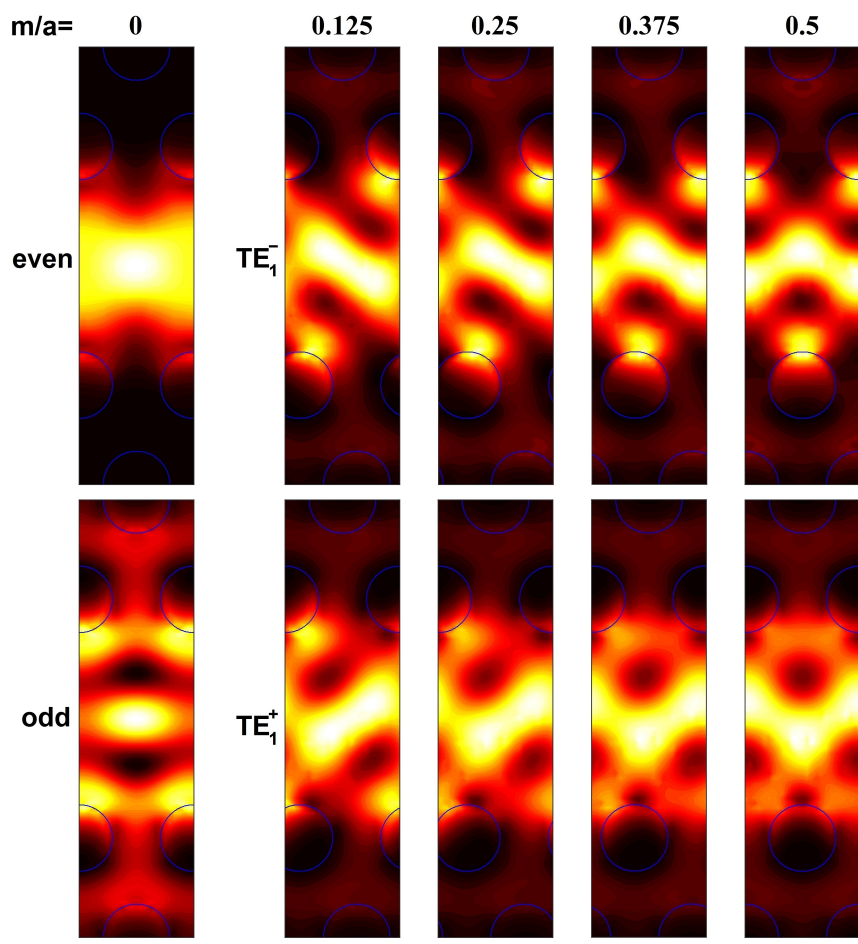

0

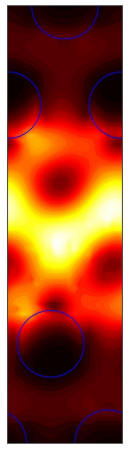

1

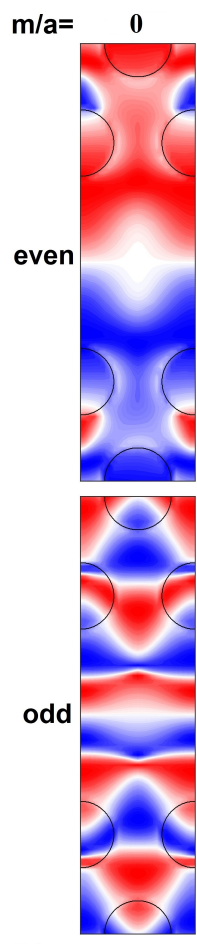

b)
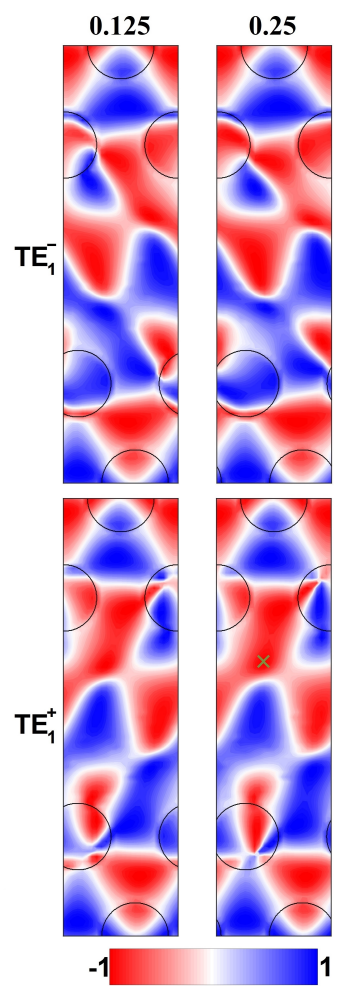

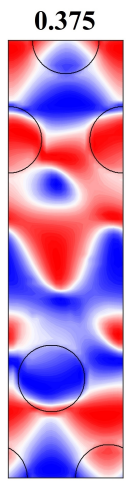

0.5

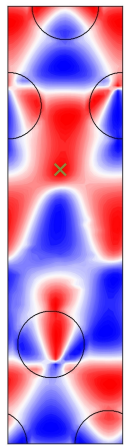

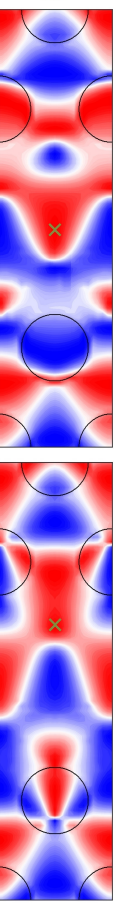

Fig. 4. The relevant properties of the odd and even mode profiles at the $k_{0}$ and of $\mathrm{TE}_{1}^{+}$and $\mathrm{TE}_{1}^{-}$modes at $k_{0}^{+}$and $k_{0}^{-}$respectively. The mismatch parameter $m / a$ has been scanned between 0 and 0.5 in steps of 0.125 . (a) The normalized Stokes Parameter $S_{0, \text { norm }}(\vec{r})=\left(\left|E_{x}(\vec{r})\right|^{2}+\left|E_{y}(\vec{r})\right|^{2}\right) / \max \left(\left|E_{x}\right|^{2}+\left|E_{y}\right|^{2}\right)$. (b) The Stokes Parameter $S_{3}(\vec{r})$, describing the degree of elliptic polarization across the PCWs unit cell. Green crosses have been superimposed at $\left|S_{3}\right|$ position for mismatches where the anomalous ZGV points are single-mode.

Stokes parameters $S_{0}$ and $S_{3}$ are defined as:

$$
\begin{gathered}
S_{0}(\vec{r})=\left(\left|E_{x}(\vec{r})\right|^{2}+\left|E_{y}(\vec{r})\right|^{2}\right), \\
S_{3}(\vec{r})=2 \frac{\operatorname{Im}\left(E_{x}^{*}(\vec{r}) E_{y}(\vec{r})\right)}{S_{0}(\vec{r})},
\end{gathered}
$$

where $\vec{r}=(x, y, z=0) . S_{0}(\vec{r})$ represents the modal intensity of the electric field [Fig.??a] and $S_{3}(\vec{r})$ characterizes the in-plane polarization ellipticity [Fig.??b]. Linear and diagonal polarization points are represented by $S_{3}=0$, while $S_{3}=1(-1)$ represents a fully right(left)-handed polarized electric field. Due to the conservation of the global chirality: $S_{3, \vec{k}}=-S_{3,-\vec{k}}$, optical transition of a QE with a specific handedness (i.e. spin dependent) occurring at $\left|S_{3}\right|=1$ will choose a wave-vector $(\vec{k})$ rather than its opposite $(-\vec{k})$.

From the symmetry perspective, the space group of a nonsymmorphic structure does not have the form of a direct product between point and translation groups. The symmetry class of the non-symmorphic PCWs could be relatively complex with respect to the value of $m / a$. For instance, if $m$ is not commensurable with $a$, the structure cannot be homomorphic to the symmorphic PCW[? ]. Although the transition between symmorphic and non-symmorphic waveguides results in the discontinuity of many physical properties of the guided modes, due to the bonding nature of the PBS, it is relevant to compare them with the odd and even modes. So, their field patterns have been also calculated at $k_{0}$.

Fig.??b points out that, in the symmorphic PCWs, circular polarization points in the core of the waveguide are prevented by the mirror symmetry. Unfortunately, the fields are also mainly concentrated in the central region for the same reason. In contrast, the replacement of the mirror symmetry with the glideplane symmetries makes this limitation obsolete. It is therefore reasonable to expect the local chirality extent to be significant at $k_{0}^{-}$and $k_{0}^{+}$, exactly where the convolution of the even and odd modes is supposed to be the most prominent. By increasing $m / a$, the modal intensities and local chirality patterns morph into undulated shapes, due to the symmetry class of non-symmorphic PCWs (Eq.??). The maximum field intensity region is stretched within the unit cell to respect the glide-plane transformations $\hat{G}_{m}$ and $\hat{G}_{m-a}$, until it splits into two for $m / a=0.5$. Because the PBS results from the bonding of modes with different guidance mechanisms and orthogonal dominant polarizations, it follows that their field profiles radically change. For instance, gap guided modes are well known to have a modal field distribution penetrating several rows of the $\mathrm{PhC}$, while index guided modes are principally concentrated in the line defect. Therefore, it can be noted that the PBS exhibit electric field intensity distributions mixing both the eigen-mode patterns in the symmorphic structure. A similar observation can be made on the elliptic polarization patterns [Fig.?? b]. The PBS mainly take the aspect of the gap guided mode outside the line defect, while their outlines inside the line defect are more complicated and fall into the scope of the bonding phenomenon.

The overlap of high intensity and circular polarization is evident for both PBS. To quantify this, a green crosses indicating $\left|S_{3}\right|=1$ have been superimposed on each ellipticity pattern where the anomalous $\mathrm{ZGV}$ points are single-mode. If 
the $\mathrm{TE}_{1}^{-}$mode at $k_{0}^{-}$is a single-mode $\mathrm{ZGV}$ point for a longer mismatch, this mode seems to display a strong confinement inside the core of the PCW. For $m / a=0.5$, the circular polarization location features a normalized electric field intensity $S_{0, \text { norm }}=S_{0} / \max \left(S_{0}\right)=0.85$. In addition, the calculated ellipticity of the maximum modal intensity $\left(S_{0, \text { norm }}=1\right)$ is $\left|S_{3}\right|=0.96$, corroborating a strong penetration of the local chirality into high field region for this mode.

On the other hand, the anomalous ZGV point $k_{0}^{+}$offers a singlemode operation from $m / a=0.25$, but the uni-directionality locations have normalized field intensities between 0.27 and 0.33 . However, the overlaps between modal intensity and local chirality patterns are indeed appreciable. For $m / a=0.5$, the maximum intensity field point has an ellipticity described by $\left|S_{3}\right|=0.78$, while extremely high intensity and nearly unitary directionality are substantiated by points displaying $\left|S_{3}\right|=0.97$ and $S_{0, \text { norm }}=0.92$.

To conclude this section, the confined anomalous ZGV points, resulting from the bonding of modes with orthogonal dominant polarizations, can exhibit circular polarization points in high field intensity regions.

\section{CONCLUSION}

To summarize, we presented in this work guided photonic states in non-symmorphic PCWs that combine confined singlemode ZGV points with circular polarization points which have the potential to demonstrate out-performing uni-directional emission. Instead of the general method to work in the vicinity of the band-edge, the slow-down effect has been forced to exist inside the BZ. This was reliant on the implementation of an effective repulsive coupling between photonic modes, with different parities, orthogonal dominant polarization components and group velocities of opposite signs. The transversal mirror symmetry has been made obsolete for inducing a branch exchange between these modes. This ensures ZGV points to exist inside the BZ which prevent the local chirality of the modes from vanishing.

Through transmission measurements on fabricated devices, the repulsive coupling strength has been measured and the singlemode anomalous ZGV points were identified by the appearance of a sub-BG contrasting with their multi-modal signature.

By using the Stokes parameters, it has been shown that the coupling of these modes, with perpendicular dominant electric field components, may be beneficial for the purpose of implementing in-plane circular polarization. The simulated fields patterns also predicted that the circular polarization points can overlap high electric field intensity regions. In particular, the $\mathrm{TE}_{1}^{-}$mode at $k_{0}^{-}$for $m / a=0.5$ where circular polarization points have been calculated to have a modal intensity $S_{0, \text { norm }}=0.85$. Hence, QE embedded in the presented PCW platform has the potential to demonstrate strong enhancement of the light-matter interaction with chiral behavior.

On top of that, we believe that the understanding of the formation of these bonding states was summarized to inspire future works in a broad range of domains, not only QED but also alloptical and electro-optic based devices. Notably, the ultra-strong photonic coupling strength, measured to be $6.74 \mathrm{THz}$ centered at $191.38 \mathrm{THz}$ for $m / a=0.5$, can also serve to tailor the bandstructure of guided modes. This magnitude is much stronger than what has been obtained in other purely photonic systems such as coupled cavities[? ] and could be used as the starting point to architect photonic devices where particular tailoring of the dispersion curve is required. The mismatch can be used to balance the even and odd components of the modes along a hybrid band like other dispersion compensation methods[? ? ? In a more general way, it is imperative for photonic integration to conceive how to establish a purely photonic coupling through the engineering of the structure, especially in monolithic integrated circuits as this work reports. It will be beneficial for the community in many aspects to consider glide-plane axis symmetry as a new degree of parametrization and take into account systematic studies of this symmetry during the design of $\mathrm{PhC}$ based devices.

\section{APPENDIX A FABRICATION}

Following our design parameters, in order to observe the signature of the anomalous ZGV points in our different PCWs, we fabricated them on a 220nm thick $<100>$ silicon on insulator wafer by the following major steps. First, the wafer was spincoated with 260nm thick layer of ZEP 520A, a positive e-beam resit. Using e-beam lithography, the patterns were exposed onto the resist, which was then developed with the use of the ZED-N50 developer, and subsequently transferred to the Si layer in an inductively coupled plasma etcher using $\mathrm{SF}_{6} / \mathrm{CHF}_{3}$ gas chemistry. The remaining resist was removed by an $\mathrm{O}_{2}$ plasma asher and the surface was cleaned further by fuming nitric acid. Continuously, the sample was dipped in a diluted Hydro-Fluoric acid (HF) to remove native oxide. Then, we spin-coated the sample using a $1.8 \mu \mathrm{m}$ thick layer of PolyMethyl-MethAcrylate (PMMA) and windows above the PhC regions were transfered by e-beam lithography. Immediately after the development of the resist in a 1:1 mixed solution of isopropyl alcohol and methyl-isobutyl-ketone, the sample was immersed in HF to remove the Burried Oxide (BOx) underneath the Si layer and suspend only the PCWs regions. Thereupon, the PMMA cap was removed by a $\mathrm{O}_{2}$ plasma asher and the sample was finally dipped in HF to end up with our designed PCWs slabs as shown Fig.??.

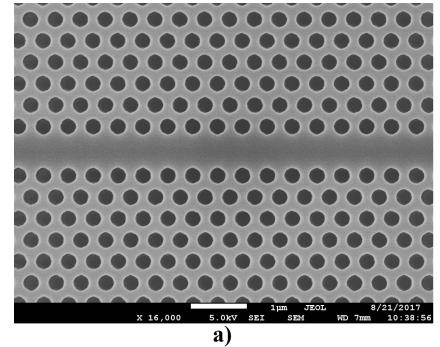
a)

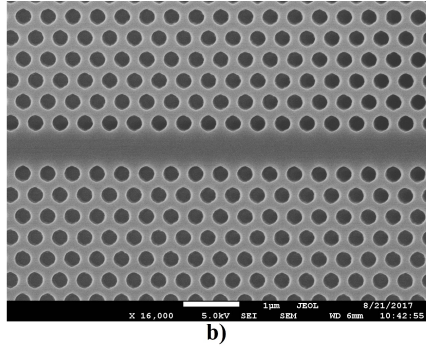

b)
Fig. 5. Images taken by scanning electron microscopy of the fabricated devices: (a) no mismatch and (b) $m / a=1 / 2$.

\section{APPENDIX B MEASUREMENT SET-UP}

The transmission spectra were measured by using a fiber coupler set-up. The light is originating from a tunable laser source, with a wavelength range from $1530 \mathrm{~nm}$ to $1610 \mathrm{~nm}$ that completely covers the simulated window of interest. Before reaching the PCWs, the light is coupled to the PhC region using fiber-grating couplers. To ensure the light was TE polarized, it passed through a fiber polarization controller and the gratings were carefully designed to maximize the transmission of this polarization. The wavelength was scanned using the built-in sweeping capability of the laser and the photo-detector automatically recorded 
each output spectrum. We performed all the measurements by using a $10 \mathrm{dBm}$ laser power. The waveguide transmissions were then normalized by setting the background loss (i.e. setup and coupling loss) to $0 \mathrm{~dB}$ using a waveguide without the $\mathrm{PhC}$ region.

\section{Funding.}

This work is supported by EPSRC Manufacturing Fellowship (EP/M008975/1), EPSRC Standard Grant (EP/M009416/1), EU FP7 Marie-Curie Carrier-Integration-Grant (PCIG13-GA-2013618116), and the University of Southampton Zepler Institute Research Collaboration Stimulus Fund.

\section{Acknowledgement.}

The authors would like to acknowledge Daniel Burt, Prof. Harvey Rutt and Prof. Satoshi Iwamoto for their fruitful discussions.

\section{Dataset.}

The data from the paper can be obtained from the University of Southampton ePrint research repository: http:/ /DOI:10.5258/SOTON/D0245.

\section{REFERENCES}

1. J. D. Joannopoulos, S. G. Johnson, J. N. Winn, and R. D. Meade, Photonic crystals: molding the flow of light (Princeton university press, 2011).

2. H. Nakamura, Y. Sugimoto, K. Kanamoto, N. Ikeda, Y. Tanaka, Y. Nakamura, S. Ohkouchi, Y. Watanabe, K. Inoue, H. Ishikawa et al., "Ultra-fast photonic crystal/quantum dot all-optical switch for future photonic networks," Opt. express 12, 6606-6614 (2004).

3. T. Yoshie, A. Scherer, J. Hendrickson, G. Khitrova, H. Gibbs, G. Rupper, C. Ell, O. Shchekin, and D. Deppe, "Vacuum rabi splitting with a single quantum dot in a photonic crystal nanocavity," Nature 432, 200-203 (2004).

4. K. Hennessy, A. Badolato, M. Winger, D. Gerace, M. Atature, S. Gulde, S. Falt, E. L. Hu, and A. Imamoglu, "Quantum nature of a strongly coupled single quantum dot cavity system," Nature 445, 896-899 (2007).

5. M. Arcari, I. Söllner, A. Javadi, S. L. Hansen, S. Mahmoodian, J. Liu, H. Thyrrestrup, E. H. Lee, J. D. Song, S. Stobbe et al., "Near-unity coupling efficiency of a quantum emitter to a photonic crystal waveguide," Phys. review letters 113, 093603 (2014).

6. I. Söllner, S. Mahmoodian, S. L. Hansen, L. Midolo, A. Javadi, G. Kiršanskè, T. Pregnolato, H. El-Ella, E. H. Lee, J. D. Song et al., "Deterministic photon-emitter coupling in chiral photonic circuits," Nat. nanotechnology 10, 775-778 (2015).

7. E. M. Purcell, Confined Electrons and Photons (Springer, 1995).

8. S. Hughes, "Enhanced single-photon emission from quantum dots in photonic crystal waveguides and nanocavities," Opt. letters 29, 26592661 (2004).

9. V. M. Rao and S. Hughes, "Single quantum-dot purcell factor and $\beta$ factor in a photonic crystal waveguide," Phys. Rev. B 75, 205437 (2007).

10. M. Burresi, R. Engelen, A. Opheij, D. Van Oosten, D. Mori, T. Baba, and L. Kuipers, "Observation of polarization singularities at the nanoscale," Phys. review letters 102, 033902 (2009).

11. A. B. Young, A. Thijssen, D. M. Beggs, P. Androvitsaneas, L. Kuipers, J. G. Rarity, S. Hughes, and R. Oulton, "Polarization engineering in photonic crystal waveguides for spin-photon entanglers," Phys. review letters 115, 153901 (2015).

12. A. Mock, L. Lu, and J. O'Brien, "Space group theory and fourier space analysis of two-dimensional photonic crystal waveguides," Phys. Rev. B 81, 155115 (2010).

13. B. Lang, D. M. Beggs, and R. Oulton, "Time-reversal constraint limits unidirectional photon emission in slow-light photonic crystals," Phil. Trans. R. Soc. A 374, 20150263 (2016).
14. S. Mahmoodian, K. Prindal-Nielsen, I. Söllner, S. Stobbe, and P. Lodahl, "Engineering chiral light-matter interaction in photonic crystal waveguides with slow light," Opt. Mater. Express 7, 43-51 (2017).

15. A. A. Sukhorukov, S. Ha, A. S. Desyatnikov, A. V. Lavrinenko, and Y. S. Kivshar, "Slow-light vortices in periodic waveguides," J. Opt. A: Pure Appl. Opt. 11, 094016 (2009).

16. P. Lodahl, S. Mahmoodian, and S. Stobbe, "Interfacing single photons and single quantum dots with photonic nanostructures," Rev. Mod. Phys. 87, 347 (2015).

17. A. Y. Petrov and M. Eich, "Zero dispersion at small group velocities in photonic crystal waveguides," Appl. Phys. Lett. 85, 4866-4868 (2004)

18. M. Ibanescu, S. Johnson, D. Roundy, C. Luo, Y. Fink, and J. Joannopoulos, "Anomalous dispersion relations by symmetry breaking in axially uniform waveguides," Phys. review letters 92, 063903 (2004).

19. M. Ibanescu, S. G. Johnson, D. Roundy, Y. Fink, and J. Joannopoulos, "Microcavity confinement based on an anomalous zero group-velocity waveguide mode," Opt. letters 30, 552-554 (2005).

20. M. Notomi, A. Shinya, K. Yamada, J.-I. Takahashi, C. Takahashi, and I. Yokohama, "Structural tuning of guiding modes of line-defect waveguides of silicon-on-insulator photonic crystal slabs," IEEE J. Quantum Electron. 38, 736-742 (2002).

21. M. Notomi, K. Yamada, A. Shinya, J. Takahashi, C. Takahashi, and I. Yokohama, "Extremely large group-velocity dispersion of line-defect waveguides in photonic crystal slabs," Phys. Rev. Lett. 87, 253902 (2001).

22. L. O'Faolain, S. A. Schulz, D. M. Beggs, T. P. White, M. Spasenović, L. Kuipers, F. Morichetti, A. Melloni, S. Mazoyer, J.-P. Hugonin et al., "Loss engineered slow light waveguides," Opt. express 18, 2762727638 (2010).

23. L. H. Frandsen, A. V. Lavrinenko, J. Fage-Pedersen, and P. I. Borel, "Photonic crystal waveguides with semi-slow light and tailored dispersion properties," Opt. Express 14, 9444-9450 (2006).

24. J. Li, T. P. White, L. O'Faolain, A. Gomez-Iglesias, and T. F. Krauss, "Systematic design of flat band slow light in photonic crystal waveguides," Opt. express 16, 6227-6232 (2008).

25. M. Qiu, M. Mulot, M. Swillo, S. Anand, B. Jaskorzynska, A. Karlsson, M. Kamp, and A. Forchel, "Photonic crystal optical filter based on contra-directional waveguide coupling," Appl. Phys. Lett. 83, 51215123 (2003).

26. W. Liu, D. Yang, G. Shen, H. Tian, and Y. Ji, "Design of ultra compact alloptical xor, xnor, nand and or gates using photonic crystal multi-mode interference waveguides," Opt. \& Laser Technol. 50, 55-64 (2013).

27. G. Milione, H. Sztul, D. Nolan, and R. Alfano, "Higher-order poincaré sphere, stokes parameters, and the angular momentum of light," Phys. review letters 107, 053601 (2011).

28. C. Bradley and A. Cracknell, The mathematical theory of symmetry in solids: representation theory for point groups and space groups (Oxford University Press, 2010).

29. S. Haddadi, P. Hamel, G. Beaudoin, I. Sagnes, C. Sauvan, P. Lalanne, J. A. Levenson, and A. Yacomotti, "Photonic molecules: tailoring the coupling strength and sign," Opt. Express 22, 12359-12368 (2014). 